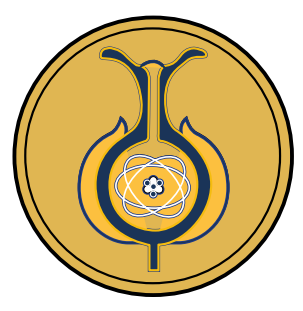

\title{
La implementación de los programas de estudio de Matemática en primaria desde la visión de la persona docente
}

\author{
Implementation of the Mathematics curriculum in elementary school from the \\ teacher's perspective
A implementação dos programas de estudo da Matemática no Ensino Fundamental a partir da perspectiva do docente

\section{Ronny Gamboa-Araya ${ }^{1}$, Randall Hidalgo-Mora ${ }^{1}$, Mario Castillo-Sánchez}

Received: Feb/4/2021 • Accepted: Aug/14/2021 • Published: Jan/31/2022

\section{Resumen (10)}

Este artículo tiene como objetivo analizar, desde la perspectiva del personal docente de primaria, los procesos que este lleva a cabo en las aulas en los aspectos metodológicos y de evaluación en el área de Matemática, como parte del desarrollo de los programas de estudio de la disciplina. El estudio se enmarca en el enfoque mixto, con un diseño secuencial. El muestreo utilizad o fue no probabilístico por conveniencia. En una primera etapa de la investigación, se encuestó a 117 docentes de educación primaria de centros educativos de Costa Rica. En la segunda etapa, se realizó una entrevista a tres docentes. Para la recolección de los datos se utilizó un cuestionario y se realizaron entrevistas semiestructuradas. Los resultados obtenidos muestran que la implementación de los programas de estudio de Matemática provocó en el personal docente cambios metodológicos y ajustes en la evaluación, pero también generó dificultades. La pizarra continúa siendo el recurso más utilizado, pero se ha diversificado el uso de otros adicionales. Hay poco uso de la tecnología y la historia de la matemática como recurso didáctico. No hay un conocimiento adecuado de los programas de estudio, se prioriza en conocimientos y no necesariamente en el desarrollo de habilidades. El personal docente posee dificultad para planificar y desarrollar actividades de resolución de problemas. Además, existe una necesidad, por parte del profesorado, de participar en procesos de formación continua en áreas como conocimientos matemáticos, estrategias metodológicas y de evaluación, uso de la tecnología, resolución de problemas, entre otras.

Palabras clave: metodología; evaluación; matemática; educación primaria; programas de estudio; docente

\section{Abstract (10)}

The objective of this paper is to analyze, from the perspectives of elementary school teachers, the processes they carry out in their classrooms in terms of methodologies and evaluations used in the development of programs for the study of mathematics. A mixed approach was used in the study, which was based on a

1 Escuela de Matemática, Universidad Nacional, Heredia, Costa Rica.

Ronny Gamboa-Araya, \ronny.gamboa.araya@una.ac.cr, (D) https://orcid.org/0000-0002-9531-0372

Randall Hidalgo-Mora, $\square$ randall.hidalgo.mora@una.ac.cr, (D) https://orcid.org/0000-0002-8405-1600

Mario Castillo-Sánchez, \ mario.castillo.sanchez@una.ac.cr, (1) https://orcid.org/0000-0002-2078-0628 
sequential design. Non-probabilistic sampling was used for convenience. In the first phase of the research, 117 elementary school teachers from schools in Costa Rica were surveyed. In the second phase, interviews were conducted with three teachers. A questionnaire was used to collect data, and semi-structured interviews were also carried out. The results obtained show that implementation of the Mathematics curriculum led to methodological changes and adjustments in the process of evaluation among the teaching staff, but also created problems. In general, the blackboard was the most used resource, but the use of additional tools has diversified. There is little use of technology and discussion of the history of mathematics as teaching resources. Knowledge of the curriculum is not adequate; priority is given to content, rather than to the development of skills. The teaching staff has difficulty in planning and developing problem-solving activities. Teachers should participate in continuous training processes in areas including mathematical knowledge, methodological and evaluation strategies, use of technology, and problem solving.

Keywords: methodology; evaluation; mathematics; elementary education; study programs; teacher

\section{Resumo}

Este artigo tem como objetivo analisar, a partir da perspectiva de professores do ensino fundamental, os processos realizados em sala de aula nos aspectos metodológicos e avaliativos na área da Matemática, como parte do desenvolvimento dos programas de estudos da disciplina. 0 estudo está enquad rado na abordagem mista, com um desenho sequencial. A amostra utilizada foi não probabilística por conveniência. Na primeira etapa da pesquisa, foram entrevistados 117 professores do ensino fundamental de centros educacionais da Costa Rica. Na segunda etapa, foi realizada uma entrevista com três professores. Para a coleta de dados, foi utilizado um questionário e realizadas entrevistas semiestruturadas. Os resultados obtidos mostram que a implementação dos programas de estudo da Matemática provocou alterações metodológicas e adequações na avaliação do corpo docente, mas também gerou dificuldades. A lousa continua sendo o recurso mais amplamente utilizado, porém, o uso de outros adicionais se diversificou. Há pouco uso da tecnologia e da história da matemática como recurso didático. Não existe um conhecimento adequado dos programas de estudo, é priorizado no conhecimento e não necessariamente no desenvolvimento de competências. 0 corpo docente tem dificuldade em planejar e desenvolver atividades de resolução de problemas. Além disso, há necessidade, por parte dos professores, de participarem de processos de formação contínua em áreas como conhecimento matemático, estratégias metodológicas e de avaliação, uso de tecnologia, resolução de problemas, entre outras.

Palavras-chave: metodologia; avaliação; matemática; ensino fundamental; programas de estudo; professor

\section{Introducción}

En mayo del 2012 el Consejo Superior de Educación de Costa Rica aprobó nuevos programas de Matemática para toda la educación primaria y secundaria del país.
Su distribución entre el personal docente se inició en el año 2013.

Según Ruiz (2013), el elemento global que motivó con fuerza la búsqueda de una reforma fue la actitud social de rechazo y temor hacia la matemática, lo cual limita las condiciones socioafectivas del 
estudiantado, padres y madres de familia, e incluso docentes, durante el proceso de enseñanza y aprendizaje de la disciplina. De acuerdo con Ruiz (2013),

Este currículo busca el dominio de conocimientos y la generación de habilidades en torno a los mismos, pero a la vez y de manera central, la construcción de capacidades transversales matemáticas que se alcanzan en el mediano y largo plazo: de razonamiento y argumentación, de representación, de comunicación, de resolución de problemas, de conexión. (Ruiz, 2013, p. 31)

El programa de estudio aprobado en el 2012, a criterio de Ruiz (2013), se diseñó con una integración vertical del primer grado escolar de la educación primaria al último de la educación secundaria, con una fundamentación teórica igual para todo el currículo, donde se mantienen las áreas matemáticas. Al respecto, Alfaro, Alpízar, Morales, Ramírez y Salas (2013) mencionan que los programas de estudio se organizan por medio de cinco áreas matemáticas; en cada área se persigue el desarrollo de habilidades específicas que se definen como capacidades a generar en plazos cortos de tiempo y que se busca integrar en la acción de aula; se asume el desarrollo de la competencia matemática como la capacidad de poder usar la matemática en diversas situaciones de la vida; se incluyen como procesos matemáticos centrales razonar y argumentar, plantear y resolver problemas, comunicar, conectar y representar. La mediación pedagógica juega un papel fundamental.

Además, Ruiz (2013) indica que se establecen cinco ejes curriculares: resolución de problemas, contextualización activa, potenciación de actitudes y creencias positivas, uso inteligente de tecnologías y uso de historia de la matemática. Las habilidades se generan a través de procesos graduales y de manera integrada en cada área que compone la malla curricular, donde los llamados procesos matemáticos de razonar $\mathrm{y}$ argumentar, plantear y resolver problemas, comunicar, conectar y representar se consideran acciones transversales (Ruiz, 2013).

Esta reforma generó, además, una serie de retos y evidenció una gama de deficiencias (Ruiz, 2013). Una de ellas fue la débil formación docente de primaria en el área de Matemática. La formación inicial en matemática de los docentes de primaria ha sido cuestionada por los pocos cursos que se dedican en las universidades a esta área.

En este sentido, Chaves, Alfaro y Alpízar (2015a) indican que no es suficiente la cantidad de cursos de matemática para que el personal docente en formación adquiera los conceptos teóricos de la asignatura; ni mucho menos poseen el conocimiento para desarrollar estos contenidos de tal forma que favorezcan el proceso de enseñanza y aprendizaje, tal como se requiere en los actuales programas de estudio.

De acuerdo con Chaves et al. (2015a), el docente debe estar capacitado para favorecer la adquisición de los conocimientos matemáticos y la aplicación de estos conocimientos en la vida cotidiana. Para lo cual se requiere una formación formal disciplinar, didáctica, metodológica, psicológica y evaluativa.

En este sentido, Chaves, Alfaro y Alpízar (2015b) señalan, con respecto al profesorado, que a nivel de primaria hay un reconocimiento de la importancia de mejorar la enseñanza de la matemática, que existe una valoración positiva sobre la reforma realizada por el MEP y que está en disposición de recibir capacitación para llevar a cabo esta labor. Además, el cuerpo docente reconoce 
que la formación universitaria fue deficiente para cumplir con los retos que plantean los nuevos programas, y se evidenciaron serias limitaciones para diseñar y planificar actividades didácticas que propicien el aprendizaje de los conceptos matemáticos.

Al respecto, y como complemento a la información anterior, Alpízar-Vargas y Alfaro-Arce (2019) realizaron una descripción del desarrollo que se da al área de las matemáticas y a su didáctica en los programas de formación profesional de los docentes de I y II ciclos de la educación general básica de Costa Rica. Las autoras, después de analizar distintos programas de formación docentes tanto en universidades públicas como privadas, señalan que:

a) En Costa Rica no existe un programa estandarizado de formación de docentes para la educación primaria, por lo que existe una amplia variedad en la oferta a nivel universitario.

b) Solamente los planes de formación de docentes de primaria de las universidades estatales cuentan con la acreditación por parte del Sistema Nacional de Acreditación de la Educación Superior (SINAES); sin embargo, este aspecto para la contratación por parte del MEP no es obligatorio.

c) La mayor parte de los centros educativos no pone a disposición de los estudiantes, de manera abierta en un sitio web, aspectos básicos del programa de formación como la estructura curricular y los descriptores de cada curso.

d) La cantidad de cursos en los programas de formación en matemática y su enseñanza es muy limitada y varía dependiendo de la universidad.

e) No se establece un perfil con respecto al personal docente encargado del proceso de formación profesional del profesorado de primaria, por lo que cada universidad decide si el docente del curso debe ser especialista en la asignatura a desarrollar. Esto implica que en los cursos relacionados con el área de Matemática no necesariamente los docentes poseen formación en esta asignatura.

f) Las universidades encargadas del proceso de formación docente en primaria están realizando esfuerzos por mejorar este con respecto al conocimiento matemático y didáctico de la asignatura; sin embargo, no existe un balance entre ambos, pues en algunas se enfatiza en lo segundo con respecto al primero.

En concordancia con lo anterior, Alpízar y Alfaro (2020), al indagar sobre la percepción de un grupo de docentes de primaria con respecto a la formación matemática recibida en su experiencia universitaria, reportan que, independiente del tipo de universidad (privada o pública), los participantes señalan que esta ha sido insuficiente para afrontar los retos educativos actuales, y citan como razones de esto la cantidad de años transcurridos desde su proceso de formación universitaria, cambios en el currículo, y que no se le ha dado la debida importancia a la actualización relacionada con los conocimientos necesarios para desempeñarse en esta asignatura.

Por lo tanto, la educación matemática en primaria se ha visto como un reto que debe ser atendido desde diferentes aspectos, que van desde la formación inicial de la persona docente hasta la práctica cotidiana en las aulas. Desde la implementación de los actuales programas de estudio se ha enfatizado en el cambio de una metodología y 
evaluación tradicional hacia nuevas estrategias basadas en resolución de problemas.

Dada esta situación, este artículo tiene como objetivo analizar, desde la perspectiva del personal docente de primaria, los procesos que se llevan a cabo en las aulas en los aspectos metodológicos y de evaluación en el área de Matemática, con la idea de aportar insumos para la elaboración de estrategias orientadas a brindar una atención integral a cuerpo docente que impacte, positivamente, su labor en el aula. Esta investigación se enmarcó en el contexto anterior a la situación sanitaria del covid-19.

Es importante aclarar que los programas de estudio de Matemática no contienen estrategias de evaluación específicas ni propusieron un cambio en la evaluación, pues no era un fin de estos. Sin embargo, resulta de interés profundizar en este aspecto para analizar la congruencia con lo establecido por el MEP y el cambio metodológico que implicó.

\section{Marco teórico}

\section{Enseñanza de la matemática en la educación primaria}

La enseñanza y aprendizaje de la matemática ha tenido siempre un puesto clave en el ámbito educativo, y actualmente se ha fortalecido con el énfasis en el desarrollo de competencias y, sobre todo, el enfoque en la resolución de problemas. Según Silva (2008), actualmente el proceso de enseñanza hace hincapié en la transmisión de los procesos de pensamiento propios de la matemática, más que en la transferencia de contenidos. Es así como la disciplina se concibe, sobre todo, como el saber hacer, y se enfatiza en los procesos mentales de resolución de problemas.
Desde este punto de vista, la metodología de resolución de problemas debería estar presente en las distintas actividades y situaciones didácticas que se presenten en el aula. En este sentido, Silva (2008) apunta que la resolución de problemas debe ser una actividad privilegiada para introducir al estudiantado en las formas propias del quehacer de la matemática, de tal forma que desarrolle procesos de pensamiento eficaces en la resolución de verdaderos problemas.

Brousseau (2000), citado por Silva (2008), "sugiere que los maestros trabajen en formular, esquematizar, visualizar problemas basados en la realidad próxima, relacionar y encontrar semejanzas entre los mismos conceptos u otras áreas del conocimiento" (Silva, 2008, p. 23). Se debe procurar el desarrollo de las competencias necesarias para el aprendizaje de la matemática, entre ellas argumentar, saber, representar, comunicar, resolver, usar técnicas e instrumentos matemáticos y modelizar; sin embargo, se reconoce que algunos miembros del personal docente aún no las desarrollan personalmente ni en su práctica.

Durante el proceso de enseñanza aprendizaje de la matemática en primaria las personas estudiantes desarrollan su capacidad de pensamiento lógico, y es aquí donde aprenden a explorar la realidad para luego representarla, explicarla y cambiarla. Por esta razón, la enseñanza de la matemática "en la educación primaria ha estado fuertemente determinada por dos tipos de reflexiones: las relativas al nivel de desarrollo intelectual y de competencia cognitiva de los alumnos y las relativas a la estructura interna del conocimiento matemático" (Godino, Batanero y Font, 2003, p. 89).

Según Godino et al. (2003) en la enseñanza primaria el proceso de construcción del conocimiento matemático debe partir de 
la propia experiencia práctica del estudiantado. Las relaciones entre las propiedades de los objetos y de las situaciones que las personas discentes establecen de forma intuitiva, pueden generar experiencias matemáticas; sin embargo, estas experiencias suelen ser meramente intuitivas y están relacionadas a la manipulación de objetos concretos.

Se espera que el estudiantado desarrolle, a lo largo de sus años de educación primaria, competencias matemáticas tales como resolver problemas de manera autónoma, comunicar información matemática, validar procedimientos y resultados y manejar técnicas eficientemente (Rodríguez, 2010). Es decir, las personas discentes de primaria deberían poder identificar, plantear y resolver, en forma autónoma, situaciones problema que se presentan en la vida cotidiana, y estar en la capacidad de comunicar los resultados y llegar a realizar generalizaciones.

Otro elemento para considerar es la formación del docente, la cual es un proceso continuo que se da a lo largo de la vida; que no se limita al conocimiento, actitudes y destrezas desarrolladas en las aulas universitarias. En este sentido, la formación de docentes ha sido un tema de interés en el sistema educativo, lo cual se ha evidenciado en distintas publicaciones y actividades académicas donde se analiza la problemática que se presenta y se generan propuestas de mejoramiento (Gonzaga, 2005).

Al respecto, Gaete y Jiménez (2011) al referirse a la educación matemática costarricense, señalan, entre otros aspectos, que un gran porcentaje del personal docente de I y II ciclos del sistema educativo público tiene problemas para establecer estrategias de mediación pedagógica en el aula para la enseñanza de la matemática, debido a una formación que no logra vincular la didáctica específica de la asignatura con el manejo de contenidos particulares; además, esta población recibe pocas capacitaciones y asesorías.

De acuerdo con los autores, esto, en parte, ha causado que la matemática pierda sentido para el estudiantado, porque la perciben desvinculada de la realidad; que se estudie memorísticamente y que las personas discentes tengan serios vacíos en contenidos matemáticos de primaria, situación que se presenta por la escasa formación que reciben muchos miembros del personal docente de I y II ciclos. Aunado a lo anterior, señalan que los recursos didácticos con los que se cuenta para la clase de Matemática son básicamente pizarra, tiza, fotocopias de prácticas y, en unos pocos casos, libros.

La Reforma de la Matemática en Costa Rica pretendió solventar algunas de las debilidades del sistema educativo y generar una serie de propuestas metodológicas enfocadas en la resolución de problemas y contextualizando la matemática con la realidad. Para estos cambios se requería de un personal docente con conocimientos matemáticos sólidos y con capacidad de innovación para poder realizar procesos metodológicos orientados a ejecutar transformaciones educativas.

Sin embargo, se debe reconocer que la mayoría de las ofertas académicas que ofrecen las universidades no presentan de manera clara el perfil de egreso de sus graduados. Puede deducirse que el personal docente desarrolla en sus años de formación inicial los conocimientos mínimos que le servirán para el análisis, la planificación, investigación y evaluación de los programas de estudio de I y II ciclos que establece el MEP (Alfaro y Alpízar, 2015).

Sin embargo, en el caso específico de Matemática, se ha destacado que la preparación inicial de los docentes de primaria 
es deficiente en esta área, principalmente propiciado por una formación de carácter generalista que no estaría acorde con la reforma educativa planteada, pues se requiere de profesionales con mayores competencias didáctico-matemáticas (Ruiz, 2013). Por esta razón, ha sido necesario, y lo es actualmente, "la implementación de procesos alternativos de formación como: congresos, capacitaciones u otros, que le permitan actualizarse y, al mismo tiempo, contrarrestar sus deficiencias cognitivas permitiéndole adecuarse a programas de estudio contextualizados y coherentes con las ideologías pedagógicas propuestas" (Alpízar et al., 2012, p. 118).

"El rendimiento académico en Matemáticas es el resultado del efecto combinado de procesos de formación docente, prácticas pedagógicas, planes y programas de estudio y contextos económicos, sociales y culturales diferenciales" (Gaete y Jiménez, 2011, p. 94). En este sentido, Fornacier (1983), citado por Gaete y Jiménez (2011), señala que para que el personal docente enseñe matemática es necesario saber matemática; por ello "y previo a toda otra condición, el maestro debe tener alguna competencia y comprensión del contenido y de las habilidades asociadas al mismo; debe saber, también, lo que significa trabajar en matemática" (p. 9).

Sobre este último aspecto, Castro (2009) indica que el personal docente de primaria debe conocer, al menos, los conceptos de cada uno de los contenidos de Matemática de todo su nivel laboral, y que para ello se le debe ofrecer un espacio donde amplíe sus conocimientos de la matemática, desarrolle el carácter formal y lingüístico que se presenta en ella, así como el lenguaje general. Desde esta perspectiva, el autor señala que el cuerpo docente debe conocer los contenidos matemáticos básicos de todo el nivel de primaria según los programas vigentes.

\section{Lineamientos de los programas de es- tudio de Matemática para primaria}

Los programas de estudio de Matemática, aprobados en el año 2012, tienen como enfoque principal la resolución de problemas, con énfasis en contextos reales y señalan cuatro momentos centrales en el desarrollo de una clase: propuesta de un problema, trabajo estudiantil independiente, discusión interactiva y comunicativa, y clausura o cierre (MEP, 2012).

La finalidad primordial de este currículo se centra en el dominio de conocimientos matemáticos por parte del estudiantado y el desarrollo de capacidades con base en esos conocimientos que le permitan, no solo su dominio, sino ser capaz de integrarlos y aplicarlos en la resolución de situaciones de la realidad. Dentro de estos programas de estudio dichas capacidades son consideradas como un eje central, y se hace una diferenciación entre aquellas que se pretende lograr en un corto plazo, llamadas "habilidades específicas", y aquellas que corresponden a la generalización de esas habilidades específicas, llamadas "habilidades generales". Además, cada una de estas habilidades está vinculada con alguna de las cinco áreas matemáticas en las que se organiza la malla curricular: Números, Medidas, Geometría, Relaciones y Álgebra, y Probabilidad y Estadística (MEP, 2012).

En los programas de estudio de Matemáticas del MEP (2012) se definen procesos matemáticos como "actividades cognitivas (o tipos de actividades) que realizan las personas en las distintas áreas matemáticas y que se asocian a capacidades para la comprensión y uso de los conocimientos" (p. 24). 
Además, añade que la incorporación de este tipo de procesos en los momentos adecuados de la lección favorecerá el desarrollo de las competencias matemáticas en diversos ámbitos; dichos procesos son: razonar y argumentar, plantear y resolver problemas, comunicar, conectar y representar (MEP, 2012).

La incorporación de los procesos matemáticos en el trabajo de aula hace necesario proponer al estudiantado situaciones con diferentes niveles de dificultad, pues "existe una relación directamente proporcional entre niveles de complejidad y las oportunidades para realizar procesos matemáticos y nutrir el progreso de la competencia matemática" (MEP, 2012, p. 32).

En este aspecto, los programas de estudio proponen tres niveles de complejidad: reproducción, conexión y reflexión, que orientan al cuerpo docente sobre el tipo o nivel de dificultad de las actividades de aprendizaje propuestas en el aula.

Según Alvarado y Vargas (2020), el docente debe considerar un énfasis curricular basado en la resolución de problemas, es decir, no se debe enfocar solamente en el nivel de reproducción, como tradicionalmente se ha trabajado. La idea es buscar un equilibrio adecuado de todos los niveles de complejidad en los problemas planteados.

Además, los programas de estudio proponen la incorporación de cinco ejes disciplinares: resolución de problemas, contextualización activa, el uso de la tecnología, actitudes y creencias y la historia de la matemática, que apoyan el proceso de enseñanza y aprendizaje en los diferentes niveles educativos.

De acuerdo con el MEP (2012), la evaluación de los aprendizajes debe considerar los siguientes principios: ser parte integral del proceso de enseñanza y aprendizaje, constituir un proceso colaborativo, valorar la pertinencia con las actividades de mediación, garantizar la congruencia de las técnicas e instrumentos de evaluación, tomar decisiones a partir de los resultados de la evaluación, y promover el compromiso hacia el aprendizaje.

Un aspecto importante en el proceso de enseñanza y aprendizaje es la evaluación. En este sentido, debe ser vista, en forma integral, como un proceso que recopila información válida y confiable para determinar el nivel de logro de las habilidades, destrezas o competencias propuestas en los programas de estudio (MEP, 2012).

\section{Metodología}

El presente estudio se enmarca en el enfoque mixto, que, de acuerdo con Hernández, Fernández y Baptista (2014), implica la recolección y el análisis de datos cuantitativos y cualitativos, su integración y discusión conjunta, para realizar hallazgos producto de toda la información recabada y lograr un mayor entendimiento del fenómeno bajo estudio.

Desde el enfoque mixto, se trabajó un diseño secuencial, pues "En una primera etapa se recolectan y analizan datos cuantitativos o cualitativos, $y$ en una segunda fase se recaban y analizan datos del otro método" (Hernández, et. al., 2014, p. 547). Este caso fue del tipo cuantitativo-cualitativo, que consistió en una etapa de recolección de información descriptiva, por medio del cuestionario, para su posterior análisis de datos y obtención de resultados, y la profundización de estos a través de entrevistas semiestructuradas.

La investigación pretendió describir el grado de conocimiento del cuerpo docente de los programas de estudio, la valoración que realizan de este, su implementación y 
metodología utilizada en la clase y el proceso de evaluación que realiza.

El muestreo utilizado para el estudio es no probabilístico por conveniencia, el cual, como explican Otzen y Manterola (2017), consiste en crear muestras de acuerdo con la facilidad de acceso y la disponibilidad de las personas de formar parte de la muestra, en un intervalo de tiempo dado.

Aunque el estudio no permite hacer inferencias para toda la población, McMillan y Schumacher (2005) apuntan que la investigación basada en muestreos no probabilísticos son los más comunes en la investigación educativa, y el uso de este tipo de muestra no significa que los resultados no sean valiosos, pues permiten un acercamiento a lo que podría suceder con la población total, así como lograr un entendimiento de las relaciones que puedan existir para un determinado fenómeno de estudio. Además, McMillan y Schumacher (2005) indican que los resultados obtenidos de estos tipos de estudio no deben descartarse y, por el contrario, deben interpretarse como válidos para poblaciones similares.

En una primera etapa de la investigación participaron 117 docentes de educación primaria de centros educativos de Costa Rica, de los cuales $85,0 \%$ aproximadamente eran mujeres, $76 \%$ tenía edad entre los 25 y 50 años, y la mayoría contaba con más de diez años de servicio (79,0\%).

El personal docente participante pertenecía a las siguientes direcciones regionales: Aguirre (5), Alajuela (14), Cañas (23), Cartago (12), Heredia (18), San Carlos (1), Nicoya (23), Occidente (2), Puntarenas (10), Puriscal (1), Sarapiquí (4), Zona Norte Norte (1) y no respondieron (3).

Aproximadamente, 96,0\% del personal docente indicó haber impartido lecciones de Matemática en educación primaria en los últimos cinco años, lo que indica que el profesorado consultado en este estudio estaba familiarizado con los programas de estudio actuales de Matemática. Respecto al tipo de institución, 97,0\% aproximadamente laboraba en escuelas públicas y $58,1 \%$ tenía la condición de profesorado propietario.

La mayoría $(80,6 \%)$ contaba con la categoría profesional de PT6 y 12,0\% con el PT5, ubicando al profesorado de este estudio en las más altas categorías profesionales según el MEP. El título universitario de mayor grado académico que poseían en educación era la licenciatura $(51,3 \%)$ o grado de maestría $(38,5 \%)$, y la mayoría $(66,7 \%)$ obtuvo este título en una universidad privada.

En la segunda etapa, con el propósito de caracterizar las prácticas metodológicas y evaluativas empleadas por el personal docente de primaria para la enseñanza de la disciplina y profundizar en los resultados obtenidos con los datos cuantitativos, se realizó una entrevista a tres docentes. Todas las participantes fueron mujeres y habían participado en la etapa anterior. Para efectos del documento las llamaremos Ana, Vivian y Rosa -no corresponden a los nombres reales de las participantes-.

Con respecto a las docentes, trabajaban únicamente en instituciones públicas y en labores de docencia, poseían nombramiento en propiedad y habían impartido lecciones de Matemática en educación primaria en los últimos cinco años.

Ana tenía 53 años, mientras que Vivian y Rosa tenían 46 años. En relación con los años de experiencia como docentes en la enseñanza primaria, poseían 26, 18 y 25 años, respectivamente. Dos de ellas pertenecían a la dirección regional de educativa de Alajuela y una de ellas a la de Puntarenas.

Todas poseían la categoría profesional en educación primaria de PT6, y su 
mayor grado académico en educación era la licenciatura. Ana y Rosa la obtuvieron en una institución pública, mientras que Vivian en una universidad privada. Con respecto a los niveles que impartían, Ana y Vivian únicamente daban lecciones a sexto grado; mientras que Rosa a tercero y quinto grado.

Como se señaló, para la recolección de los datos se utilizó un cuestionario, el cual fue aplicado por correo electrónico y en forma presencial, en algunas regiones educativas, en el II semestre del 2019. Se contó con una base de direcciones electrónicas de docentes de diferentes direcciones regionales; y la aplicación presencial fue en reuniones convocadas por la Dirección Regional de Educación.

La construcción del cuestionario se basó fundamentalmente en los programas de estudio de Matemática. Fue validado por medio de juicio de expertos (cinco expertos en el área de la Educación Matemática y Estadística) y mediante una prueba piloto.

Dicho cuestionario estaba formado por 5 módulos: una primera parte de información general de los docentes, una segunda dedicada específicamente a los programas de estudio de Matemática del MEP, un tercer módulo referente a la formación continua del docente, una cuarta parte dedicada a los aspectos metodológicos presentes en los programas de estudio, y la última sección referida a los aspectos propiamente de evaluación. Para el análisis de las respuestas del cuestionario se obtuvieron los porcentajes de respuesta para cada pregunta según las categorizaciones establecidas para cada ítem.

Para las entrevistas semiestructuradas se realizó una guía basada en los datos cuantitativos obtenidos, con el propósito de profundizar en estos. Esta guía de entrevista fue validad por tres expertos en Educación
Matemática. La información obtenida se analizó con base en las categorías definidas para el cuestionario.

\section{Análisis y resultados}

\section{Conocimiento de los programas de estudio de Matemática}

Un aspecto consultado al profesorado fue el conocimiento y uso de los programas de estudio de Matemática del MEP. Primero, ante la pregunta sobre el medio por cual consultan el documento, la mayoría del personal participante indicó que lo hace de manera digital $(53,8 \%)$ o a través del documento impreso $(35,9 \%)$, por lo que un alto porcentaje tiene acceso a leerlo y examinarlo. Aunado a lo anterior, 42,0\% del profesorado indicó consultar los programas propuestos por el MEP al menos una vez al mes, y 13,0\% indica que lo revisa todos los días.

Sobre la frecuencia mensual con que consultan los programas de estudio para la preparación de sus lecciones, las docentes entrevistadas indican hacerlo una o dos veces al mes, para su programación mensual, y revisan no solo los conocimientos sino las habilidades que se pretende desarrollar; en algunas ocasiones, consultan la fundamentación teórica. En el caso de Rosa, ella los consulta, especialmente, para revisar los conocimientos que debe desarrollar; no presta tanta importancia a los otros aspectos que se contemplan en él.

Los programas de estudio no solamente se pueden utilizar como consulta para ciertas secciones específicas, sino que cuentan con otra información que es relevante y que debe ser de conocimiento del personal docente para dar integralidad al proceso educativo. 
En la tabla 1 se puede observar que el profesorado indica que los ítems o secciones de mayor consulta en los programas de estudio son las habilidades específicas, conocimientos $\mathrm{y}$ orientaciones metodológicas propuestas.

Con respecto al enfoque de resolución de problemas propuesto por el MEP en los programas de estudio para lograr el aprendizaje en los estudiantes, el profesorado manifestó en su mayoría $(79,8 \%)$ que los problemas formulados en la clase deben pertenecer al contexto del estudiantado. En este sentido, 58,8\% manifestó que los problemas planteados deben llevar a cada miembro del alumnado a un reto intelectual e involucrar conceptos que no conoce todavía.

Tabla 1.

Distribución del profesorado según el aspecto que más consulta en los programas de estudio de Matemática del MEP, 2019

\begin{tabular}{lcc}
\hline \multicolumn{1}{c}{ Ítems } & Frecuencia & Porcentaje \\
\hline Habilidades específicas & 83 & 72,8 \\
$\begin{array}{l}\text { Orientaciones } \\
\text { metodológicas propuestas }\end{array}$ & 65 & 57,0 \\
$\begin{array}{l}\text { Conocimientos o } \\
\text { contenidos }\end{array}$ & 65 & 57,0 \\
Habilidades generales & 55 & 48,2 \\
$\begin{array}{l}\text { Orientaciones para la } \\
\text { evaluación }\end{array}$ & 51 & 44,7 \\
Indicaciones puntuales & 40 & 35,1 \\
Propósitos de enseñanza & 39 & 34,2 \\
Fundamentos de los & 14 & 12,3 \\
programas & & \\
\hline Fur Pras
\end{tabular}

Fuente: Propia de la investigación.

Con el propósito de profundizar en el conocimiento que tenía el personal docente participante sobre los programas de estudio, se realizaron varias preguntas enfocadas en las áreas de conocimiento matemático, los niveles de complejidad y ejes disciplinares. En la tabla 2 se muestran las respuestas del personal docente respecto a las áreas de conocimiento matemático.
Estas áreas, según los programas de estudio, son las siguientes: medidas, números, geometría, relaciones y álgebra, y estadística y probabilidad; es importante mencionar que a pesar de que la mayoría del profesorado indica conocer los programas de estudio, señala áreas de conocimiento que no están incluidas en él, como análisis $(47,8 \%)$, aritmética $(45,2 \%)$ y resolución de problemas $(61,7 \%)$. Lo anterior podría indicar que no existe un conocimiento real de los programas de estudio. Un docente afirmó que no conoce estas áreas.

\section{Tabla 2.}

Distribución del profesorado según áreas de conocimiento que está contemplado en los programas de estudios del MEP, 2019

\begin{tabular}{lcc}
\hline \multicolumn{1}{c}{ Categorías } & Frecuencia & $\begin{array}{c}\text { Porcentaje } \\
\text { de casos }\end{array}$ \\
\hline Geometría & 106 & 92,2 \\
Medidas & 103 & 89,6 \\
Relaciones y álgebra & 102 & 88,7 \\
Estadística y & 101 & 87,8 \\
probabilidad & & \\
Resolución de problemas & 71 & 61,7 \\
Análisis & 55 & 47,8 \\
Aritmética & 52 & 45,2 \\
No las conozco & 1 & 0,9 \\
\hline
\end{tabular}

Fuente: Propia de la investigación.

En cuanto a los niveles de complejidad en los cuales se deben ubicar los problemas y actividades que se plantean en el aula, propuestos en los programas de estudio de Matemática del MEP, se definen los siguientes: reflexión, conexión y reproducción. En la tabla 3 se observa que, al consultarle al profesorado sobre estos y su conocimiento, un alto porcentaje no tiene claros los niveles de complejidad; además, importante mencionar que el $11 \%$ aproximadamente indica que no los conoce. 
Tabla 3.

Distribución del profesorado según niveles de complejidad que está contemplado en los programas de estudio del MEP, 2019

\begin{tabular}{lcc}
\hline \multicolumn{1}{c}{ Categorías } & Frecuencia & $\begin{array}{c}\text { Porcentaje } \\
\text { de casos }\end{array}$ \\
\hline Reflexión & 83 & 73,5 \\
Representación & 68 & 60,2 \\
Ejemplificación & 57 & 50,4 \\
Reproducción & 56 & 49,6 \\
Conexión & 55 & 48,7 \\
Abstracción & 34 & 30,1 \\
No los conozco & 12 & 10,6 \\
\hline
\end{tabular}

Fuente: Propia de la investigación.

Respecto a los ejes disciplinares, se le solicitó al profesorado que seleccionara de una lista todos aquellos que los programas de estudio de matemática proponían y que acompañan el proceso de enseñanza. Los ejes disciplinares contemplados por el MEP son actitudes y creencias, uso de tecnología, contextualización activa, resolución de problemas e historia de la matemática. El propósito fue identificar si el personal docente podía identificar los correspondientes.

En la tabla 4 se muestra el porcentaje de respuesta para algunos de los ítems propuestos, indicados por el profesorado, en donde mencionan algunas que no corresponden a los ejes disciplinares. Es evidente que los docentes dan un mayor énfasis a la resolución de problemas, con 79,5\%; además, el $62,5 \%$ de los docentes menciona la resolución de ejercicios como eje disciplinar, el cual no es uno de ellos. Esto podría ser un indicador de que muchos de ellos consideran la "resolución de problemas" y "resolución de ejercicios" como lo mismo o algo similar. Sobre esta idea debe profundizarse en futuras investigaciones.
Tabla 4.

Distribución del profesorado según ejes disciplinares que indican que están contemplados en los programas de estudio del MEP, 2019

\begin{tabular}{lcc}
\hline Ítem o eje disciplinar & Frecuencia & $\begin{array}{c}\text { Porcentaje } \\
\text { de casos }\end{array}$ \\
\hline Resolución de problemas & 89 & 79,5 \\
Resolución de ejercicios & 70 & 62,5 \\
Uso de tecnología & 67 & 59,8 \\
Historia de la & 66 & 58,9 \\
matemática & & \\
Aptitudes y habilidades & 59 & 52,7 \\
Aplicación de & 57 & 50,9 \\
habilidades & & \\
Contextualización activa & 43 & 38,4 \\
Actitudes y creencias & 29 & 25,9 \\
No los conozco & 6 & 5,4 \\
\hline
\end{tabular}

Fuente: propia de la investigación.

En los programas de estudio se contemplan como procesos matemáticos razonar y argumentar, plantear y resolver problemas, comunicar, conectar y representar. Según el MEP, a estos se les debe dar énfasis durante las lecciones de Matemática. Al personal docente participante se le solicitó que escribiera los tres procesos matemáticos a los que regularmente daba mayor énfasis durante sus lecciones, y mencionó como procesos matemáticos que guían sus lecciones los siguientes: formulación, tratamiento y solución de problemas; problemas de análisis; razonamiento matemático; lectura y comprensión; actividades de inicio, reflexión, actividades de cierre; practicas individuales; contextualización; habilidades; problemas iniciales; construcción del conocimiento; evaluación formativa; propuesta del problema; discusión interactiva; trabajo colectivo, construcción del conocimiento y resolución de casos. Lo anterior muestra que el profesorado realmente no tiene claros los lineamientos que propone el MEP para la preparación de las lecciones y desconoce los procesos matemáticos. 
Adicional a los programas de estudio, el MEP brinda un documento auxiliar titulado Distribución de conocimientos y habilidades especificas para la implementación de los programas de estudio de matemática para I y II ciclos de la Enseñanza General Básica, según año y período escolar. El documento tiene como propósito brindar a quienes imparten educación primaria la distribución de los conocimientos y habilidades específicas para cada uno de los años escolares, según los periodos lectivos; y realiza una estimación del total de lecciones requeridas para el desarrollo de las habilidades, así como para el "Aprendizaje de conocimientos" y "Movilización y aplicación de los conocimientos".

Este documento constituye una ayuda adicional en la planificación del proceso educativo y es parte de los documentos adicionales que pone a disposición el proyecto de la Reforma de la Educación Matemática en Costa Rica. Sin embargo, 41,9\% del profesorado participante manifestó no conocerlo. Del porcentaje del personal docente que indica conocerlo, solo $50,0 \%$ de ellos y ellas lo utilizó para el planeamiento diario de sus lecciones. Un aspecto que se puede destacar con base en la entrevista realizada a las docentes es que ellas también señalan no conocer este documento.

Este documento plantea indicaciones que guían el proceso de planeamiento de la clase en aspectos como desarrollo lineal de todas las habilidades de cada área de conocimiento, mezcla de habilidades de las diferentes áreas a lo largo del año lectivo, grupos de habilidades que se deben trabajar de manera integrada, total de lecciones que se requieren para abordar las habilidades, cantidad de lecciones que deben dedicarse a cada etapa, número de lecciones que se deben utilizar para la evaluación y habilidades que deben reforzarse durante todo el año.

Sin embargo, el profesorado que conoce el documento indica que los tres principales aspectos que se incluyen en este son las habilidades que deben reforzarse durante todo el año $(67,7 \%)$, mezcla de habilidades de las diferentes áreas a lo largo del año lectivo $(64,5 \%)$ y grupos de habilidades que se deben trabajar de manera integrada $(54,8 \%)$. Los otros aspectos no surgen como relevantes.

Otra consulta que se le realizó al profesorado fue la relacionada con la distribución del tiempo para abarcar todos los programas de estudio. Ante esta interrogante, 56,4\% manifestó que no es suficiente. Es notorio resaltar que una de las quejas más frecuentes del profesorado es el no poder abarcar los programas de estudios por diversas razones, tanto curriculares como no curriculares.

\section{Valoración de los programas de es- tudio de Matemática}

Aproximadamente 60,0\% del profesorado consultado consideró que los programas de estudio no poseen deficiencias, mientras que un 34,2\% indica que sí. Entre las observaciones realizadas sobre este último aspecto, el cuerpo docente menciona que:

a) Los programas de estudio deberían adecuarse según el contexto de los centros educativos y la capacidad de cada estudiante. En este sentido, las docentes entrevistadas señalaron que el documento debería ser menos teórico e incluir más ejemplos en diferentes contextos.

b) Los programas de estudio no están concebidos para la población con dificultades o deficiencias en su aprendizaje; el modelo evaluativo hace que algunas personas discentes pasen al siguiente ni- 
vel sin dominar aspectos importantes.

Al respecto, las docentes entrevistadas apoyan la idea de que los actuales programas de estudio no están diseñados para incluir a toda la población estudiantil, pues no consideran a los estudiantes con dificultades de aprendizaje ni las distintas realidades que viven los docentes en las aulas.

c) Los programas de estudio son demasiado extensos, es difícil abarcarlos todos de forma dinámica y son complejos de manipular, pues la información está en distintas secciones, distribuida en todo el documento. Además, las docentes entrevistadas agregan que se presentan estrategias poco atractivas o no realizables en algunos contextos.

d) En algunas habilidades no se mencionan diferentes actividades de mediación que guíen a la persona docente. Aunado a esto, las docentes entrevistadas indican que algunas habilidades son difíciles de desarrollar o no hay claridad de lo que se espera con ellas.

e) Hay escaza contextualización y conexión entre áreas, pocas sugerencias de actividades y fichas de trabajo, y baja exigencia de análisis y reflexión del estudiantado.

Con respecto a los conocimientos matemáticos, se realizó la consulta al personal docente sobre cuáles consideraba importantes para la educación matemática en primaria y que no están incluidos en los programas de estudio. La mayoría (62,3\%) indica que no se necesitan más conocimientos.

Igualmente, al profesorado se le consultó cuáles conocimientos matemáticos consideraba que no son importantes para la educación matemática en primaria y que están incluidos en los programas de estudio.
La gran mayoría de la muestra encuestada manifiesta que no quitaría conocimientos $(91,0 \%)$; por otra parte, algunos manifiestan que suprimirían los siguientes conocimientos: razones y proporciones, álgebra, cuerpos geométricos, fórmulas, divisiones con tres o cuatro cifras, medidas, números decimales en la recta, notación desarrollada, cuerpos sólidos, probabilidad y razonamiento lógico.

Sobre este último aspecto, y aunque no es el objetivo de este artículo, es importante acotar que lo manifestado por algunos docentes debe investigarse con mayor detalle. El hecho, por ejemplo, de que se sugiera eliminar el razonamiento lógico de una asignatura como Matemática podría reflejar que maneja una idea errónea sobre la disciplina, su propósito y el fin de su enseñanza. Esto podría llevar, incluso, a pensar que ciertos conocimientos por sí solos no son necesarios, sin visualizar a la materia como un todo y las habilidades que se deben desarrollar con ayuda de los conocimientos propuestos.

Con respecto a la valoración de los programas de estudio de Matemática, las docentes entrevistadas destacaron la diferencia entre los programas de estudio anteriores y los actuales. Una de las principales diferencias que citan es que los programas actuales están centrados en el desarrollo de habilidades y no en el contenido. En este sentido, no se busca dar al estudiantado muchos conocimientos y que este los reproduzca, sino que se fomenta el desarrollo progresivo de ciertas habilidades. Aunado a esto, mencionan que con los programas actuales se pretende dejar de lado la clase magistral para dar oportunidad a la persona discente de que interactúe con su conocimiento y lo construya por medio de actividades de resolución de problemas. 
Además, indican que el papel del cuerpo docente cambió, pues antes era el "dueño" de la clase; mientras que ahora el protagonista del proceso educativo es el alumnado. Se pretende, en este sentido, que la persona docente sea un guía en el proceso de aprendizaje y no un transmisor de conocimiento.

\section{Implementación de los programas de estudio de Matemática y meto- dologías en la clase de Matemática}

Con el propósito de ayudar al personal docente con la implementación de los programas de estudio, el proyecto de Reforma de la Educación Matemática en Costa Rica ha dispuesto en su sitio web material, videos y otros recursos para la planificación y desarrollo de la clase. Esta información se ha comunicado por diferentes medios y es de libre acceso para cualquier persona docente.

Respecto a la frecuencia de consulta de estos materiales para complementar las estrategias metodológicas que se emplean durante las lecciones, la mayoría del personal docente encuestado $(56,4 \%)$ manifiesta no conocerlos, $23,1 \%$ indica que los conoce, pero no los utiliza, y $14,5 \%$ los utiliza o consulta 1 o 2 veces por semana.

Un aspecto mencionado por dos de las docentes entrevistas es que ellas, para su planeamiento, sí han consultado el sitio web de la Reforma de la Educación Matemática en Costa Rica. En algunas ocasiones han visitado el sitio para consultar las actividades que se proponen para el desarrollo de las habilidades. Mencionan que no todas las actividades expuestas se pueden desarrollar con el estudiantado, pero que sí han adaptado algunas de ellas o han tomado algunas ideas para implementar en sus lecciones.

Propiamente para el desarrollo de la clase, la mayoría del personal docente encuestado indica que el desarrollo de habilidades es el principal aspecto que orienta sus lecciones. El uso de la modelización matemática no fue mencionado por ninguna persona docente. En la tabla 5 se detallan los porcentajes.

Tabla 5.

Distribución del profesorado según aspectos que orientan el planeamiento de sus clases de Matemática, 2019

\begin{tabular}{lcc}
\hline \multicolumn{1}{c}{ Ítem } & Frecuencia & $\begin{array}{c}\text { Porcentaje } \\
\text { de casos }\end{array}$ \\
\hline $\begin{array}{l}\text { Desarrollo de } \\
\text { habilidades }\end{array}$ & 58 & 52,3 \\
$\begin{array}{l}\text { Resolución de problemas } \\
\text { Objetivos de aprendizaje }\end{array}$ & 51 & 45,9 \\
$\begin{array}{l}\text { Evaluación de los } \\
\text { aprendizajes }\end{array}$ & 36 & 35,1 \\
$\begin{array}{l}\text { Resolución de ejercicios } \\
\text { Conocimientos }\end{array}$ & 35 & 32,4 \\
matemáticos & 31 & 31,5 \\
$\begin{array}{l}\text { Contextualización activa } \\
\text { Uso de tecnología como }\end{array}$ & 16 & 27,9 \\
recurso didáctico & 9 & 14,4 \\
$\begin{array}{l}\text { Aspectos históricos } \\
\text { Modelización }\end{array}$ & 3 & 8,1 \\
matemática & 0 & 2,7 \\
\hline
\end{tabular}

Fuente: Propia de la investigación.

Con respecto al planeamiento, las docentes entrevistadas consideran que este se basa completamente en los lineamientos del MEP, que el tiempo disponible es insuficiente para cubrir en su totalidad los programas de estudio y que por ello deben hacer modificaciones para desarrollar los conocimientos. En este caso particular se destaca que, aunque los programas de estudio se basan en habilidades, las docentes no las mencionan y apuntan centrarse en los conocimientos.

Adicionalmente, a estas maestras se les consultó, en forma específica, sobre cómo incentivan el desarrollo de las habilidades. El propósito fue conocer sobre actividades específicas. Las docentes señalaron 
que se basan en lo que indican los programas de estudio y en los documentos que les aportan las Asesorías Regionales. Además, utilizan la Caja de Herramientas del MEP (disponible en línea) para analizar posibles actividades y orientaciones para desarrollar en clase. Sin embargo, no hicieron una alusión directa a actividades específicas.

En este sentido, al consultarles cuáles habilidades de los programas de estudio tratan de desarrollar en sus estudiantes, ellas indican que la conexión, el razonamiento, la representación y la argumentación. Sin embargo, tampoco profundizan en la forma en que las incentivan y no mencionan otras que se incluyen en el documento del MEP.

Con respecto al desarrollo de las habilidades, las docentes entrevistadas indican que, aunque tratan de desarrollarlas en las personas estudiantes, esto no se da en forma óptima. Ana, por ejemplo, señala:

Muchas de las habilidades que están propuestas ahi pueden trabajarse con el grupo; lo que pasa es que yo personalmente considero que ese programa de estudios está diseñado para estudiantes sin dificultades académicas, y en la escuela la población a la que yo atiendo hay mucha dificultad (Ana, comunicación personal, 5 de mayo 2020).

Además, esta docente señala que los conocimientos previos de los estudiantes, ausencia de habilidades requeridas y acceso limitado a recursos para la enseñanza son aspectos que, entre otros, dificultan el desarrollo de las habilidades contempladas en los programas de estudio.

Al continuar refiriéndose a su planeamiento de la clase, Vivian y Rosa señalan, por ejemplo, que suelen centrarse en el conocimiento que deben desarrollar. Contemplan, al iniciar un tema, hacer un resumen de lo que la persona estudiante debe saber e incluyen ejemplos y prácticas para el desarrollo de la clase. Aunado a lo anterior, indican que toman en cuenta el contexto en el cual se desenvuelve el estudiantado, con el propósito de plantear actividades relacionadas con su vida diaria.

De lo señalado por las docentes entrevistadas se puede interpretar que, aunque son conscientes de la importancia del desarrollo de las habilidades, aún siguen centrándose en los conocimientos y hacen una combinación entre conocimientos $\mathrm{y}$ habilidades, sin hacer referencia a actividades específicas para su desarrollo.

Con respecto al desarrollo de la clase de Matemática y la metodología más frecuente que utiliza el profesorado para el desarrollo de esta, solamente $37,0 \%$ de las personas encuestadas manifiesta utilizar la siguiente secuencia: propuesta del problema, trabajo estudiantil independiente, discusión interactiva y comunicativa entre el estudiantado y el estudiantado-profesorado, y clausura y cierre a cargo de la persona docente, que es la sugerida por el MEP en los programas de estudio.

Sobre este respecto, las docentes entrevistadas reconocen que iniciar un tema con un problema contextualizado que propicie la construcción del conocimiento ha sido un cambio, y un reto, con la implementación de los programas de estudio. Aunque no se aplique frecuentemente, sí conocen la metodología propuesta por el MEP.

Sobre los recursos que el personal docente emplea en las clases de Matemática, se destaca que, respecto a los libros de texto, el profesorado encuestado indica que se utilizan con mayor frecuencia en los niveles superiores: sexto $(37,5 \%)$, quinto $(33,0 \%)$ y cuarto $(31,3 \%)$. El nivel donde menos se utiliza es primero, con $24,1 \%$ del profesorado. 
En cuanto a la frecuencia de uso de los libros de texto en las lecciones de Matemática, $41,0 \%$ del profesorado menciona que los utiliza siempre o casi siempre, 25,6\% los utiliza algunas veces, mientras que $3,4 \%$ indica que los usa pocas veces o nunca.

Además, respecto a otros recursos diferentes al libro de texto, el personal docente indica utilizar actividades lúdicas, material concreto, computadora, proyector multimedia, pizarra, material fotocopiado, folletos con fichas de trabajo elaboradas por el profesorado, algunos softwares, televisión, calculadora, entre otros. La lista de recursos que señaló el profesorado fue amplia.

Con respecto a los recursos tecnológicos a los cuales las personas docentes tienen acceso para usar en sus clases de Matemática, la mayoría manifiesta tener acceso a computadora portátil con conexión a internet y proyector multimedia. Los recursos menos utilizados son las tabletas electrónicas y pizarras digitales o inteligentes. Esto indica que el personal docente cuenta con los recursos o condiciones para hacer uso de las tecnologías en el aula; sin embargo, destacan que, aunque las instituciones posean estos recursos, no son suficientes para atender a toda la población o se presenta problemas de conexión.

Por otra parte, el profesorado indicó una serie de estrategias metodológicas o recursos y la frecuencia con que los emplea durante sus lecciones de Matemática. En la tabla 6 se muestran los resultados relativos al uso de estas estrategias. Es importante recordar que estos datos se recolectaron previo a la situación sanitaria del covid-19.

Es importante subrayar que las estrategias, sugeridas en el cuestionario, en su mayoría, son usadas pocas veces o algunas veces. Respecto a la construcción del conocimiento a través del uso de material concreto, ninguna persona docente lo aplica

Tabla 6.

Distribución del profesorado según estrategia metodológica y recursos empleados por nivel de uso, 2019

\begin{tabular}{|c|c|c|c|c|c|}
\hline Estrategias metodológicas o recursos & Siempre & $\begin{array}{c}\text { Casi } \\
\text { siempre }\end{array}$ & $\begin{array}{l}\text { Algunas } \\
\text { veces }\end{array}$ & $\begin{array}{l}\text { Pocas } \\
\text { veces }\end{array}$ & Nunca \\
\hline $\begin{array}{l}\text { Construcción del conocimiento a través del uso de algún } \\
\text { software o calculadora }\end{array}$ & 24,8 & 30,8 & 28,2 & 8,5 & 2,6 \\
\hline $\begin{array}{l}\text { Presentación de los contenidos a través de material } \\
\text { fotocopiado }\end{array}$ & 3,4 & 14,5 & 28,2 & 41,9 & 8,5 \\
\hline Trabajo colaborativo & 0,0 & 3,4 & 28,2 & 42,7 & 23,1 \\
\hline Aplicación de modelaje matemático & 9,4 & 19,7 & 30,8 & 23,1 & 8,5 \\
\hline Uso de la historia de la matemática & 17,1 & 26,5 & 29,9 & 11,1 & 7,7 \\
\hline $\begin{array}{l}\text { Lista de ejercicios para la interiorización de los } \\
\text { conocimientos matemáticos }\end{array}$ & 2,6 & 10,3 & 29,1 & 41,0 & 12,8 \\
\hline Elaboración de proyectos & 16,2 & 20,5 & 29,9 & 21,4 & 6,0 \\
\hline Clase magistral & 11,1 & 30,8 & 28,2 & 17,9 & 6,0 \\
\hline $\begin{array}{l}\text { Utilización de libros de texto como medio para realizar } \\
\text { práctica }\end{array}$ & 13,7 & 6,8 & 29,1 & 35,9 & 9,4 \\
\hline $\begin{array}{l}\text { Utilización de libros de texto para el aprendizaje y registro de } \\
\text { los conocimientos matemáticos }\end{array}$ & 12,0 & 13,7 & 29,9 & 29,9 & 8,5 \\
\hline Uso de situaciones problema contextualizadas & 0,9 & 1,7 & 27,4 & 46,2 & 18,8 \\
\hline $\begin{array}{l}\text { Construcción del conocimiento a través del uso de material } \\
\text { concreto }\end{array}$ & 0,0 & 2,6 & 28,2 & 47,0 & 17,9 \\
\hline Asignación de trabajo individual al estudiante & 0,0 & 1,7 & 30,8 & 40,2 & 23,9 \\
\hline
\end{tabular}

Fuente: Propia de la investigación. 
siempre, y muy pocas casi siempre. Lo mismo sucede con el trabajo colaborativo.

En este sentido, las docentes entrevistadas señalaron, con respecto al trabajo colaborativo, que han tratado de plantear una mayor cantidad de trabajos en grupo y de implementar actividades en las que puedan participar todas las personas estudiantes; lo anterior como parte de los cambios curriculares y de planeamiento didáctico.

Vivian, por ejemplo, indica que la implementación de los programas de estudio le ha implicado usar menos fotocopias y más uso de la tecnología. Rosa señala que ha tenido que disponer en su aula de recursos adicionales para que el estudiantado trabaje, tales como libros, paletas, chapas, etc., con el fin de utilizarlos en cualquier momento según sus necesidades.

Respecto a la frecuencia de uso de los recursos didácticos en el desarrollo de las clases de Matemática, es interesante observar, en la tabla 7, que la pizarra es uno de los más utilizados; mientras aquellos asociados con tecnología no se usan con regularidad.
El material concreto manipulable se utiliza pocas veces o nunca.

Respecto a las actividades de aula diseñadas y propuestas por el profesorado, se les solicitó que indicaran, según su consideración, la frecuencia en que estas incentivaban los siguientes procesos matemáticos: razonar, argumentar, plantear problemas, resolver ejercicios, comunicar, conectar, memorizar, resolver problemas y representar.

Se debe hacer la aclaración de que, de acuerdo con lo establecido por el MEP en los programas de estudio, resolver ejercicios y memorizar no son procesos matemáticos incluidos en la propuesta, pero se incorporaron para identificar si el profesorado era capaz de identificar los planteados por el MEP y si estaban dando prioridad a otros aspectos.

En la tabla 8 se muestran los resultados, donde los procesos de razonar, resolver ejercicios, comunicar y resolver problemas se incentivan pocas veces. Se destaca que memorizar, aunque no posee mayoría de respuestas, es el proceso que tiene mayor porcentaje de frecuencia de uso.

Tabla 7.

Distribución del profesorado según uso de recursos didácticos empleados por el profesorado en sus clases de matemática, 2019

\begin{tabular}{lccccc}
\hline \multicolumn{1}{c}{ Recursos didácticos } & Siempre & Casi siempre & Algunas veces & Pocas veces & Nunca \\
\hline Pizarra & 46,2 & 28,2 & 19,7 & 2,6 & 0,0 \\
Televisor & 5,1 & 12,0 & 17,9 & 6,0 & 52,1 \\
Teléfono móvil & 0,0 & 8,5 & 16,2 & 16,2 & 53,0 \\
Material fotocopiado & 21,4 & 29,1 & 35,0 & 6,0 & 2,6 \\
Calculadora & 6,8 & 8,5 & 28,2 & 21,4 & 28,2 \\
Software matemático & 0,9 & 5,1 & 12,8 & 10,3 & 64,1 \\
Proyector multimedia & 4,3 & 7,7 & 40,2 & 14,5 & 28,2 \\
Libro de texto & 18,8 & 29,1 & 24,8 & 10,3 & 12,0 \\
Guías de trabajo & 19,7 & 30,8 & 33,3 & 4,3 & 8,5 \\
Material concreto manipulable & 20,5 & 33,3 & 31,6 & 10,3 & 0,9 \\
Juegos & 15,4 & 27,4 & 42,7 & 8,5 & 1,7 \\
\hline
\end{tabular}

Fuente: Propia de la investigación. 
Tabla 8.

Distribución del profesorado según su uso de los procesos matemáticos en las actividades de aula, 2019

\begin{tabular}{lccccc}
\hline \multicolumn{1}{c}{ Procesos matemáticos } & Siempre & Casi siempre & Algunas veces & Pocas veces & Nunca \\
\hline Razonar & 0,9 & 2,6 & 16,2 & 43,6 & 32,5 \\
Argumentar & 2,6 & 4,3 & 28,2 & 35,9 & 22,2 \\
Plantear problemas & 1,7 & 6,8 & 28,2 & 32,5 & 24,8 \\
Resolver ejercicios & 0,9 & 1,7 & 9,4 & 42,7 & 41,0 \\
Comunicar & 0,9 & 5,1 & 14,5 & 45,3 & 27,4 \\
Conectar & 5,1 & 7,7 & 28,2 & 29,1 & 21,4 \\
Memorizar & 2,6 & 23,1 & 37,6 & 23,1 & 3,4 \\
Resolver problemas & 0,0 & 0,9 & 8,5 & 46,2 & 40,2 \\
Representar & 0,9 & 6,8 & 29,9 & 35,9 & 20,5 \\
\hline
\end{tabular}

Fuente: Propia de la investigación.

Al profundizar en la entrevista sobre el desarrollo de la clase y las actividades que se proponen en ella, Ana señala, por ejemplo, utilizar en algunas ocasiones artículos de periódicos o una encuesta y apoyarse en estos recursos para realizar una pregunta inicial. Luego, da un espacio para que las personas estudiantes la analicen en forma individual o en grupos.

Posterior a esta actividad, la docente selecciona a alguna persona estudiante para que exponga sus respuestas, y utiliza estas para introducir el tema y hacer las explicaciones correspondientes. Finalmente, realiza prácticas para que el alumnado aplique su conocimiento.

En contraposición, Vivian indica que primero explica la materia y, luego, utiliza fichas de trabajo, las cuales cada discente resuelve individualmente o en grupo. A partir de esta y las respuestas realizadas por el estudiantado, ella realiza una retroalimentación.

Rosa señala que ella inicia con una serie de preguntas que realiza a los estudiantes, relacionadas con los conocimientos a desarrollar, para identificar qué saben ellos. Considerando el conocimiento de estos, la docente indica que trata de iniciar con actividades motivantes, según los recursos disponibles, y con historias relacionadas con su entorno para introducir y desarrollar los conocimientos.

Yo trato como de siempre introducir con un cuento, con una historia, con una canción, algo que a ellos se les ocurra, que tenga que ver con el tema como para motivarlos, verdad. Desearía tener videos y demás... Pero yo trato de que no sea muy tediosa la ... pensando en mi enseñanza de matemáticas en aquellas épocas, trato de que sea más divertida para ellos, que no la vean como algo feo, verdad (Rosa, comunicación personal, 11 de mayo de 2020).

Sin embargo, la docente expresa que desearía contar con mayores recursos para utilizar en sus clases o que el acceso a internet en su institución fuera mejor, para investigar y emplear los recursos disponibles en línea.

Las docentes entrevistadas valoran que, en general, en sus lecciones se potencia el desarrollo de actitudes y creencias positivas hacia la matemática. Ellas mencionan desplegar una actitud positiva, implementar juegos, utilizar distintos recursos y realizar clases amenas, pero que no se logra en su 
totalidad porque hay muchos factores que afectan la enseñanza de la disciplina; entre ellos, la actitud y gusto por la disciplina y la poca motivación e interés del estudiantado.

La resolución de problemas es un eje central de los programas de estudio. Con respecto a su uso durante la clase de Matemática, Ana indica que ella la trabaja, en algunos temas, por medio de actividades lúdicas, para introducir un tópico específico. Posteriormente, propone otras actividades para que el estudiantado las realice en forma individual en sus cuadernos y emplea prácticas para reforzar los conocimientos. Sin embargo, reconoce que no siempre profundiza en el nivel requerido de una habilidad específica.

Aunque las docentes entrevistadas tratan de utilizar elementos del entorno para proponer actividades de resolución de problemas, señalan que se encuentran con dificultades para su construcción. Parte de la dificultad para plantear las actividades de resolución de problemas la indica Rosa al señalar:

No son perfectas las situaciones de problemas porque, bueno, a mí me gusta mucho ir a los congresos de Ciencia, Tecnología y Sociedad todos los años y estuve en un taller en donde ellos nos indicaban que no es cualquier situación problema la que usted presenta; uno tiene para eso que saber hacer una situación problema de acuerdo al tema, y eso tampoco lo indican ni le explican a uno esas cosas. Tiene uno que andar ahi agarrándolo (Rosa, comunicación personal, 19 de mayo 2020).

Con respecto a la contextualización y cómo esta se utiliza en el aula de Matemática, las maestras entrevistadas tratan de proponer actividades relacionadas con el contexto en el que los estudiantes se desarrollan, su entorno familiar y la comunidad en la que está inmersa la escuela.

Por otra parte, relacionado con el uso de la tecnología, indican que utilizan, aunque en pocas oportunidades, videos sobre solución de ejercicios e historia de la matemática. Entre los recursos de que señalan disponer está el proyector multimedia y la computadora, pero con uso poco frecuente, debido a la insuficiente disponibilidad de estos, la falta de tiempo para atender el grupo y desarrollar los conocimientos, y problemas con la conexión a internet. Cuando emplean la computadora, por lo general, esta corresponde a la que poseen las docentes para su uso personal.

Las tres docentes indican que en sus instituciones el estudiantado no tiene acceso a recursos tecnológicos, por lo que no se pueden plantear actividades con el uso de dichos recursos. La situación es más grave al considerar las condiciones de las personas discentes en sus hogares, pues se presentan grandes diferencias.

Con respecto al uso de la historia de la matemática, hacen poca referencia a actividades concretas y la emplean en muy pocas ocasiones. Solo ven algunos videos o realizan algunas lecturas en clase, las cuales comentan con el estudiantado, pero alegan que no despiertan el interés en él. Además, mencionan que, por falta de tiempo, no profundizan en esta temática. Incluso, Rosa señala que

Cuando hay esa parte en el programa... es algo que también es diferente... que podemos ver la historia y contarla para que ellos se emocionen; pero no es que yo use muchísimo la historia. $O$ sea, si yo tengo que contar algo lo hago así como muy por encimita, pero no la 
utilizo mucho la verdad; le soy honesta, no estoy mintiendo (Rosa, comunicación personal, 19 de mayo 2020).

Al consultarles a las entrevistadas sobre las dificultades que se han presentado con la aplicación de los programas de estudio, ellas señalan que, aunque tratan de iniciar los temas con un problema, a la hora de proponérselo al estudiantado este no muestra esfuerzo por resolverlo o entenderlo. Sigue esperando que sea la persona docente quien le dé toda la información.

La realidad de las escuelas unidocentes es un aspecto que Rosa denuncia que no se considera en los programas de estudio. El tener que atender varios grupos a la vez, así como el tamaño de estos, dificulta el tipo de actividades que se proponen y el seguimiento que se le puede dar a cada estudiante. A pesar de esto, la docente indica que hace un esfuerzo por trabajar con cada uno y cada una, explicarles y darles orientación en las actividades que ella propone en el aula.

Otro aspecto mencionado por las docentes es que la cantidad de actividades extracurriculares que se desarrollan en las instituciones y la pérdida de lecciones por otras actividades académicas dificultan desarrollar todas las acciones que se planean, lo que influye en el cumplimiento de los programas de estudio. Aunado a esto, la cantidad de adecuaciones curriculares que deben atender, y la atención personalizada de algunas de ellas, les dificultan poder desarrollar algunas de las actividades.

En general, las maestras entrevistadas reconocen que algunos miembros del cuerpo docente de primaria no están desarrollando los programas de estudio tal como están planteados, por lo que el estudiantado se ve afectado al cambiar a una persona docente que sí está tratando de aplicarlo. Esto genera diferencia no solo en la metodología que se emplea en la clase sino en la formación de las personas discentes.

Finalmente, al cuestionar a las docentes sobre su formación universitaria en el área de Matemática y cómo esta afecta su labor profesional en la aplicación de los programas de estudio, Rosa admite, por ejemplo, que la universidad no la preparó para este programa ni su planeamiento.

Las docentes alegan que la cantidad de conocimientos matemáticos que se abarcaron en sus procesos de formación son insuficientes para desarrollar los programas de estudio, los cuales exigen un gran dominio de estos. Ana, por ejemplo, describe que su formación en el área fue muy básica y esto no le ayuda para su desarrollo profesional.

Además, señalan que hay una desactualización de los conocimientos abarcados en sus procesos de formación y lo que deben desarrollar en la clase. Incluso, conocen algunos planes de formación profesional donde lo que se desarrolla en los cursos no tiene relación con la realidad de aula en la que ellas están inmersas.

\section{Estrategias de evaluación aplicadas por personas docentes}

En lo referente a la evaluación, al profesorado se le dio una serie de proposiciones para que indicara el grado de aceptividad a la hora de completar la frase "La evaluación de los estudiantes es", con las proposiciones propuestas.

En la tabla 9 se indica lo manifestado por el profesorado, donde se puede observar que para las proposiciones "una parte integral del proceso de enseñanza y aprendizaje", "una oportunidad para modificar el proceso de enseñanza y aprendizaje", "una herramienta para orientar el trabajo del alumnado" y "una herramienta para informar a 
mis alumnos sobre sus avances, progresos y aspectos que deben mejorar", ningún docente ni ninguna docente indicó estar muy de acuerdo con estas proposiciones.

Respecto al momento en que se aplica la evaluación al estudiantado, la mayoría del personal docente encuestado $(70,6 \%)$ manifiesta que lo hace durante el desarrollo del conocimiento, 50,5\% indica que al terminar el desarrollo del conocimiento, $y$, en menor porcentaje, al inicio del tema, con $23,9 \%$. Otras personas docentes manifiestan que en todas las lecciones o en el periodo de evaluaciones institucionales, con un porcentaje de $47,7 \%$ y $41,3 \%$, respectivamente.

Por otra parte, los instrumentos de evaluación más utilizados son el portafolio $(59,0 \%)$, las pruebas orales $(23,1 \%)$ y los exámenes finales (14,5\%). En la tabla 10 se muestran los resultados en detalle.

Tabla 9.

Distribución del profesorado según grado de aceptividad con frases asociadas a la evaluación del estudiantado, 2019

\begin{tabular}{|c|c|c|c|c|c|}
\hline $\begin{array}{c}\text { Proposiciones } \\
\text { La evaluación de los estudiantes es... }\end{array}$ & $\begin{array}{l}\text { Muy de } \\
\text { acuerdo }\end{array}$ & De acuerdo & $\begin{array}{l}\text { Ni de acuerdo ni } \\
\text { en desacuerdo }\end{array}$ & $\begin{array}{c}\text { En } \\
\text { desacuerdo }\end{array}$ & $\begin{array}{c}\text { Muy en } \\
\text { desacuerdo }\end{array}$ \\
\hline $\begin{array}{l}\text {...una oportunidad para reflexionar sobre } \\
\text { el valor de mi trabajo. }\end{array}$ & 3,4 & 5,1 & 18,8 & 35,0 & 29,9 \\
\hline $\begin{array}{l}\text {...un instrumento para conocer las } \\
\text { habilidades específicas alcanzadas. }\end{array}$ & 0,9 & 2,6 & 11,1 & 50,4 & 28,2 \\
\hline $\begin{array}{l}\text {...una parte integral del proceso de } \\
\text { enseñanza y aprendizaje. }\end{array}$ & 0,0 & 0,9 & 11, & 53,8 & 27,4 \\
\hline $\begin{array}{l}\text {...una distorsión del proceso de } \\
\text { aprendizaje. }\end{array}$ & 13,7 & 39,3 & 27,4 & 6,0 & 4,3 \\
\hline $\begin{array}{l}\text {...una obligación que tengo dentro de mi } \\
\text { tarea docente. }\end{array}$ & 11,1 & 17,9 & 17,9 & 23,1 & 21,4 \\
\hline $\begin{array}{l}\text {...una oportunidad para modificar el } \\
\text { proceso de enseñanza y aprendizaje. }\end{array}$ & 0,0 & 5,1 & 13,7 & 42,7 & 30,8 \\
\hline $\begin{array}{l}\text {...una herramienta para orientar el } \\
\text { trabajo del alumnado. }\end{array}$ & 0,0 & 1,7 & 13,7 & 46,2 & 30,8 \\
\hline $\begin{array}{l}\text {...un proceso para determinar la } \\
\text { aprobación o no en el nivel educativo. }\end{array}$ & 6,0 & 13,7 & 26,5 & 34,2 & 11,1 \\
\hline $\begin{array}{l}\text {...un instrumento para determinar el } \\
\text { conocimiento del estudiante. }\end{array}$ & 2,6 & 10,3 & 23,1 & 41,0 & 15,4 \\
\hline ...un proceso de carácter punitivo. & 10,3 & 23,9 & 35,0 & 16,2 & 5,1 \\
\hline $\begin{array}{l}\text {...un proceso donde la persona } \\
\text { estudiante aprende del docente y de } \\
\text { sus compañeros(as) y donde el docente } \\
\text { aprende de sus estudiantes. }\end{array}$ & 3,4 & 7,7 & 12,8 & 39,3 & 29,9 \\
\hline $\begin{array}{l}\text {...un instrumento para ajustar mi trabajo } \\
\text { docente, mis materiales y recursos } \\
\text { educativos empleados. }\end{array}$ & 1,7 & 6,8 & 16,2 & 46,2 & 22,2 \\
\hline $\begin{array}{l}\text {..un proceso que requiere de } \\
\text { información cualitativa y cuantitativa. }\end{array}$ & 0,9 & 1,7 & 12,0 & 47,9 & 28,2 \\
\hline $\begin{array}{l}\text {...una herramienta para informar a mis } \\
\text { alumnos y alumnas sobre sus avances, } \\
\text { progresos y aspectos que deben mejorar. }\end{array}$ & 0,0 & 5,1 & 11,1 & 49,6 & 24,8 \\
\hline
\end{tabular}

Fuente: Propia de la investigación. 
Tabla 10.

Distribución del profesorado según frecuencia con que utiliza los instrumentos de evaluación en las clases de Matemática, 2019

\begin{tabular}{lccccc}
\hline \multicolumn{1}{c}{ Instrumentos de evaluación } & Siempre & $\begin{array}{c}\text { Casi } \\
\text { siempre }\end{array}$ & $\begin{array}{c}\text { Algunas } \\
\text { veces }\end{array}$ & $\begin{array}{c}\text { Pocas } \\
\text { veces }\end{array}$ & Nunca \\
\hline Pruebas cortas & 12,0 & 15,4 & 29,1 & 23,9 & 10,3 \\
Cuaderno de los alumnos & 0,9 & 2,6 & 12,0 & 39,3 & 38,5 \\
Pruebas orales & 23,1 & 15,4 & 25,6 & 17,1 & 7,7 \\
Portafolios & 59,0 & 17,1 & 6,8 & 2,6 & 1,7 \\
Exámenes parciales & 12,0 & 10,3 & 8,5 & 18,8 & 42,7 \\
Trabajo en clase & 0,0 & 0,0 & 2,6 & 22,2 & 69,2 \\
Exámenes finales & 14,5 & 3,4 & 7,7 & 20,5 & 44,4 \\
Registro de autoevaluación del estudiante & 15,4 & 14,5 & 13,7 & 18,8 & 27,4 \\
Tareas & 1,7 & 2,6 & 6,8 & 28,2 & 53,0 \\
Registro de coevaluación del estudiante & 19,7 & 17,1 & 19,7 & 15,4 & 17,1 \\
Guías de observación & 6,0 & 9,4 & 17,1 & 30,8 & 26,5 \\
\hline
\end{tabular}

Fuente: Propia de la investigación.

Con respecto a la evaluación del trabajo cotidiano, al profesorado se le solicitó indicar las técnicas que utiliza para la evaluación de este. En la tabla 11 se resume la frecuencia de uso; la mayoría utiliza la lista de cotejo y las escalas de valoración.

Tabla 11.

Distribución del profesorado según frecuencia con que se utilizan los instrumentos de evaluación en el trabajo cotidiano en las clases de Matemática, 2019

\begin{tabular}{lcc}
\hline Técnicas & Frecuencia & $\begin{array}{c}\text { Porcentaje } \\
\text { de casos }\end{array}$ \\
\hline Listas de cotejo & 82 & 76,6 \\
Escalas de valoración & 69 & 64,5 \\
Listas de control & 30 & 28,0 \\
Anecdotarios & 16 & 15,0 \\
Cuestionarios & 15 & 14,0 \\
Diarios & 12 & 11,2 \\
Grabaciones y & 3 & 2,8 \\
filmaciones & & \\
Sociogramas & 1 & 0,9 \\
\hline
\end{tabular}

Fuente: Propia de la investigación.

Además de lo anterior, al personal docente se le consultó si consideraba que las técnicas que utiliza para la evaluación del trabajo cotidiano le permitían identificar el progreso real de cada estudiante, a lo que la mayoría indicó que sí $(72,6 \%)$.

Con respecto a la evaluación, las docentes entrevistadas señalan, en general, que la implementación de los programas de estudio ha implicado modificaciones. Han tratado de hacer más énfasis en el proceso y no tanto en los aspectos teóricos. Aunque resaltan que en la evaluación sumativa no se han presentado grandes cambios y que la oportunidad para realizarlos es poca.

Hay una valoración, por parte de las docentes entrevistadas, de la evaluación como un proceso que no depende únicamente de un valor obtenido a partir de resultados sumativos, y consideran que el trabajo en el aula es una importante fuente de información para analizar el progreso de cada estudiante. Con respecto al proceso de evaluación que realizan, se concluye que ejecutan procesos de evaluación diagnóstica, formativa y sumativa.

En la evaluación formativa, tratan de estar más pendientes del desarrollo de las habilidades en el estudiantado y lo basan 
principalmente en la observación del trabajo cotidiano. Cuando plantean actividades al inicio o durante la clase, tratan de hacer preguntas para que las personas discentes las contesten y así valorar su aprendizaje. En este sentido, Rosa indica:

A mí me gusta más el registro anecdótico en donde yo indico o voy observando qué es lo que más le cuesta al niño, si él logró el contenido que yo quería y, si no lo logró, qué puedo yo hacer o qué tengo que seguir haciendo en el proceso para que ese niño, en específico, pueda continuar, para obtener el conocimiento que yo deseo o que el plan me dice (Rosa, comunicación personal, 11 de mayo de 2020).

La observación en clase, por ejemplo, permite analizar el aprendizaje del estudiante desde otra perspectiva, indagar sobre este y realizar acciones correctivas en el momento. Esto, aunado a la elaboración de rúbricas y registros anecdóticos para analizar el avance del estudiantado, les permite tener una visión sobre los aprendizajes de las personas estudiantes y el desarrollo de las habilidades, así como fortalezas y debilidades.

Sin embargo, se reconoce que mantener actualizados ambos registros, en algunos casos, resulta difícil, por todas las actividades que implica el desarrollo de la clase y por la cantidad de discentes que se debe atender.

Las docentes también utilizan trabajos extraclase como un medio para reforzar las actividades y conocimientos que se desarrollan en clase, y en los aspectos que ellas consideran deben profundizarse aún más. Sin embargo, señalan que este tipo de asignaciones se utiliza poco y corresponde a tareas cortas, algunas de las cuales se deben calificar con base en rúbricas.
Además, las docentes indican que también se aplican, en algunos casos, pruebas cortas, tanto formativas como sumativas. La evaluación sumativa la identifican asociada propiamente con los exámenes. Sin embargo, reconocen que el rendimiento en este instrumento de evaluación, en particular, se ve afectado en muchos casos por los nervios de la persona estudiante.

Es importante señalar que, con respecto a las pruebas escritas, las docentes indican que no siempre les permiten determinar cuánto ha aprendido cada estudiante. Algunas personas estudiantes, por lo general, lo que hacen es memorizar a corto plazo, lo que no permite determinar un verdadero aprendizaje.

Las docentes resaltan, también, que este instrumento se ha convertido en un factor de desmotivación para muchas personas estudiantes. Rosa, al respecto, menciona:

A veces una prueba escrita lo que hace es frustrar a un estudiante. Yo he tenido estudiantes que son geniales en la clase, son increíbles tutores para otros estudiantes, pero la prueba escrita la ven como lo peor de lo peor (...) y llegan a la prueba y no hacen nada, y el padre de familia dice "Pero ¿cómo es posible? Si se sabía todo” (Rosa, comunicación personal, 19 de mayo de 2020).

De acuerdo con las docentes, la evaluación ahora enfatiza más en el proceso y no solo en la respuesta final que aporta el estudiantado. Por lo que las estrategias que se emplean deben enfocarse en aprender a aprender, y no solo en la memorización de conocimientos.

Las docentes entrevistadas señalan que, con base en los resultados de las evaluaciones, ellas analizan los errores y toman acciones correctivas. Una de estas es solicitar el apoyo de los padres y madres de familia para requerir que, fuera del horario de clase, 
se pueda profundizar en la temática estudiada; así como los servicios de apoyo de la institución, con el propósito de implementar estrategias que le permitan a cada estudiante lograr los aprendizajes. Sin embargo, reconocen que, algunas veces, en el hogar no encuentran las ayudas requeridas, por lo que siempre hay personas estudiantes que no logran alcanzar el desarrollo requerido.

Todas las docentes señalaron utilizar listas de ejercicios o prácticas como una forma de verificar si la persona estudiante está desarrollando una determinada habilidad. Sin embargo, no todos son del tipo de resolución de problemas; algunos son algorítmicos y se enfocan en el manejo de conocimientos y procedimientos, no en el desarrollo de habilidades.

Otro aspecto mencionado por las docentes es que la evaluación que sugiere el MEP no está en concordancia con lo que se incluye en los programas de estudio, lo cual limita e influye en el trabajo de aula, pues se sigue un sistema de evaluación tradicional.

\section{Capacitación y retos}

Un tema relevante en este estudio fue conocer la perspectiva del profesorado sobre su formación continua. Esta formación no solo debe ir enfocada al repaso de conocimientos específicos, sino que debe generar otras habilidades sobre su base ya existente, y crear espacios de discusión entre el profesorado sobre las metodologías empleadas en la clase y que le han dado buenos resultados.
El profesorado considera importante el acompañamiento por parte del MEP en los procesos de actualización y formación continua. La mayoría del personal docente encuestado señala que el MEP realiza periódicamente procesos de capacitación sobre los programas de estudio de Matemática.

A la consulta sobre a cuántas capacitaciones presenciales o virtuales, impartidas por el MEP (Asesorías Regionales, proyecto Reforma de la Matemática, entre otros), ha asistido y que estén relacionadas directamente con algún aspecto de los programas de estudio de Matemática, 50,0\% del profesorado manifestó nunca haber asistido o haber asistido solo a una, mientras que un $30,7 \%$ manifestó haber asistido a entre dos y cinco capacitaciones.

Con respecto a las temáticas que el profesorado considera que se deben incluir o profundizar en futuras capacitaciones, en la tabla 12 se muestran los resultados. Se observa que $75,2 \%$ del profesorado encuestado valora que la temática que se debería incluir, o profundizar con mayor detalle, es la construcción de problemas contextualizados para el desarrollo de la lección de Matemática. Se evidencia que el uso de la historia como recurso didáctico no es relevante para el profesorado, pues solamente $15 \%$ indicó

Tabla 12.

Temáticas de capacitación que se deberían incluir o profundizar en las capacitaciones impartidas a docentes de primaria por parte del MEP, 2019

\begin{tabular}{lcc}
\hline \multicolumn{1}{c}{ Temática de capacitación } & Frecuencia & Porcentaje de casos \\
\hline Problemas contextualizados & 85 & 75,2 \\
Construcción de actividades para discusión interactiva & 77 & 68,1 \\
Actitudes y creencias & 72 & 63,7 \\
Estrategias de evaluación del trabajo realizado en el aula & 66 & 58,4 \\
Construcción de actividades para el trabajo estudiantil & 53 & 46,9 \\
Planificación de las dos etapas en la lección de Matemática & 52 & 46,0 \\
Uso de la tecnología como recurso didáctico & 44 & 38,9 \\
Fundamentos teóricos de los programas de Matemática & 29 & 25,7 \\
Ejemplo de uso de la historia como recurso didáctico & 17 & 15,0 \\
Construcción de actividades para cierre o clausura & 1 & 0,9 \\
\hline
\end{tabular}

Fuente: Propia de la investigación. 
que se debe incluir o profundizar sobre este tema en las capacitaciones, si bien también se señaló que es un recurso poco empleado.

Respecto a los contenidos matemáticos en los cuales el profesorado señala requerir-capacitación, tenemos que relaciones y álgebra, geometría y estadística y probabilidades son los temas más recurrentes. En cuanto a la modalidad de las capacitaciones, la mayoría $(54,7 \%)$ la prefiere de forma presencial, $28,2 \%$ indica que bimodal y $12,8 \%$ que de manera virtual.

Adicionalmente, las docentes entrevistadas consideran que se debería recibir capacitación en evaluación, abordaje de las adecuaciones curriculares, estrategias didácticas, fundamentación teórica del programa de estudios y uso de la tecnología. Además, destacan que deberían ser tipo taller, donde el personal docente pueda hacer, proponer, discutir y aprender a plantear actividades para desarrollar en el aula. No deben ser capacitaciones teóricas donde el profesorado solo se dedique a escuchar.

Al personal docente encuestado se le consultó, además, sobre el número de capacitaciones recibidas en los últimos cinco años en el uso de recursos tecnológicos aplicados a la enseñanza de la matemática; la mayoría $(55,6 \%)$ indica que no ha recibido capacitación, $32,5 \%$ ha recibido entre una y tres capacitaciones en dicho periodo y $10,3 \%$ manifiesta que ha recibido cuatro o más capacitaciones en el último quinquenio.

La implementación de los programas de estudio también implicó una serie de retos. Las docentes entrevistadas señalan que trabajar con estos les implicó estudiar, por su cuenta, algunos de los conocimientos matemáticos y consultar libros y documentos que les permitieran profundizar no solo en el conocimiento de la disciplina sino en el componente pedagógico. Otro aspecto en el que tuvieron que profundizar fue en la comprensión de las habilidades a desarrollar; elemento sobre el cual consideran aún deben ahondar más.

Un elemento que continúa siendo un reto es el planteamiento de las situaciones problema, pues se les dificulta su construcción, según las habilidades requeridas, y que, a la vez, resulten motivantes para el estudiantado.

La mejora de algunos aspectos relacionados con el estudiantado es un reto que el profesorado debe asumir. En este sentido, las docentes entrevistadas señalan que existe, en forma generalizada, una mala actitud, creencias previas equivocadas e indiferencia hacia la materia. Además, señalan algunas deficiencias que poseen las personas discentes como malos hábitos de estudio, poca compresión lógica, falta de análisis y razonamiento, dificultad en el paso de lo concreto a lo abstracto, ausentismo y poco interés en su aprendizaje. Lo anterior, aunado en muchos casos al poco compromiso de los encargados de las personas discentes, entorpece el proceso de aprendizaje.

Finalmente, las docentes mencionan, sobre el proceso educativo en sí, que, en general, este debería ser más práctico y con menos evaluaciones. Lo extenso de los programas de estudio y la dificultad para su desarrollo se ven agravados por la inversión excesiva de tiempo en asuntos administrativos (informes, adecuaciones, comisiones, entre otras), que resta tiempo a la planificación y preparación profesional.

\section{Conclusión}

Los resultados de la investigación nos permiten delinear algunos hallazgos referentes a la implementación de los programas de estudio y a aspectos metodológicos 
y evaluativos de la educación matemática en primaria, según los instrumentos aplicados a la muestra docente.

Existe un porcentaje importante del profesorado que no posee un conocimiento adecuado de los programas de estudio en aspectos como procesos matemáticos, habilidades, metodología de resolución de problemas y áreas matemáticas. Por ende, no se puede aspirar a que la implementación de lo estipulado por el MEP se esté llevando a cabo en forma adecuada.

En este sentido, y aunque la mayoría del personal participante señaló tener acceso al documento de los programas de estudio, pareciera que no se ha realizado una lectura y análisis de este en forma detallada; a la vez, este debe ser fuente de consulta regular. Aunque se reconoce que los programas de estudio realmente son una guía en el proceso de educativo, se señala que muchas personas docentes no los utilizan, y basan sus lecciones en la programación de un libro de texto.

Esta situación es un aspecto que se debe estudiar a profundidad para analizar sus implicaciones, pues podría suceder que los libros de texto empleados por parte del personal docente no estén totalmente vinculados con los programas de estudio; además, podría limitar la propuesta y el desarrollo de actividades orientadas al desarrollo de las habilidades en el estudiantado.

Con respecto a la valoración de los programas de estudio de Matemática, se reconoce que hay diferencias con el anterior en aspectos como planificación y desarrollo de la clase, metodología, papel de la persona estudiante y del profesorado; la enseñanza ahora se basa en habilidades y no en conocimientos. Sin embargo, existe un porcentaje de docentes que siguen enfatizando en el conocimiento matemático.
Aunque en aspectos evaluativos no hubo cambios en los programas de estudio, el profesorado señala que sí los han tenido que realizar para ser consecuentes con lo expuesto por el MEP.

La mayoría del personal docente manifiesta estar de acuerdo con el enfoque de resolución de problemas propuesto por el MEP en los programas de estudio, como un medio para lograr el aprendizaje en los estudiantes; aspecto que coincide con lo indicado por Chaves et al. (2015b) respecto a que existe una valoración positiva sobre la reforma realizada por el MEP.

El profesorado, además, indica que los problemas deben pertenecer al contexto del estudiante, que deben llevar a este a un reto intelectual e involucrar conceptos que no conoce todavía. Sin embargo, hay dificultades para su puesta en práctica a nivel de aula.

Por otra parte, hay una consideración de que los programas de estudio no contemplan las necesidades educativas de una parte del estudiantado ni los diferentes contextos en los cuales se desarrolla el proceso educativo, aunque uno de sus ejes es la contextualización activa.

Para las personas docentes la implementación de los programas de estudio les ha implicado una mayor atención al trabajo de cada estudiante, quien es el actor principal del proceso educativo, a su desarrollo y sus necesidades educativas. También requirió la inclusión en la clase de actividades de resolución de problemas contextualizadas que permitiera a la persona discente la construcción de su conocimiento, por medio de un proceso de participación activa en este. Esto acompañado de un mayor uso de la tecnología, mayor cantidad de actividades de trabajo en grupo, uso de material concreto, videos, entre otros. 
Parte del personal docente ha complementado su clase con distintos recursos como fichas de trabajo, la pizarra, material concreto, material desechable, manipulativos, entre otros. La pizarra es uno de los recursos más utilizados; mientras que, en general, la tecnología y la historia de la matemática no se utilizan regularmente como recursos didácticos. En sus lecciones, el personal docente continúa empleando listas de ejercicios como una forma de incentivar en cada estudiante la comprensión de los conocimientos y el manejo de procedimientos. Lo datos anteriores concuerdan con lo expuesto por Gaete y Jiménez (2011) al referirse a la educación matemática costarricense a nivel de primaria.

Se visualiza un esfuerzo por parte del personal docente por implementar y realizar cambios en su práctica educativa orientados al desarrollo del programa de estudios y sus fundamentos. Tratan de crear ambientes de aprendizaje donde la persona discente se sienta motivada y aprecie la matemática.

Se pudo observar que hay un esfuerzo por aplicar la resolución de problemas y la contextualización activa. Sin embargo, el profesorado afronta dificultades en la planificación, planteamiento y desarrollo de este tipo actividades. En muchos casos, la resolución de problemas se asume como sinónimo de problemas matemáticos o ejercicios algorítmicos. Esto podría incidir en el aprendizaje del estudiantado y afectar el desarrollo de habilidades en esta área, lo que no permitiría lograr este objetivo de la educación matemática en primaria, de acuerdo con Rodríguez (2010).

En el proceso de implementación de los programas de estudio se han presentado varias dificultades. Una de ellas, como se señaló, ha sido la asociada a la formulación de actividades de resolución de problemas, pues ha sido un proceso difícil y que requiere de capacitación para profundizar en este tipo de tareas.

Otra dificultad ha sido la actitud de parte del estudiantado frente a este tipo de actividades, pues no está acostumbrado a trabajar con ellas, se desmotiva y prefiere, muchas veces, que sea la persona docente quien resuelva las situaciones y las explique, mientras ellos y ellas se mantienen en un papel pasivo.

Aunado a lo anterior, mencionan que el tiempo disponible para cubrir los programas de estudio, tal como están formulados, es insuficiente, pues hay factores propios de la institución que afectan el desarrollo de las lecciones, como la pérdida de clases y actividades extracurriculares; además de aspectos académicos y la disponibilidad de recursos económicos y tecnológicos.

Aunque se reconoce que los programas de estudio se basan en el desarrollo de habilidades, se continúa mencionando como una prioridad y guía del proceso educativo el desarrollo de los conocimientos. Lo anterior muestra que, aunque se tiene claro, a una parte del personal docente se le dificulta planificar sus lecciones con base en el desarrollo de habilidades, donde los conocimientos son un medio para ello. Posiblemente, es una prioridad heredada de los programas de estudio anteriores que aún se mantiene.

Incluso, sobre las habilidades que se plantean en los programas de estudio se señala que algunas de ellas son difíciles de desarrollar o no se vinculan directamente con el conocimiento matemático y que, además, aspectos como la deficiencia en conocimientos previos y ausencia de recursos para la enseñanza dificultan este proceso.

Hay un sector que indica que los conocimientos que posee en la materia no son suficientes para desempeñar su labor, y es 
un aspecto que puede incidir en cómo se está desarrollando el proceso de enseñanza y aprendizaje. Este resultado concuerda con lo señalado por Chaves et al. (2015a), Chaves et al. (2015b), Alpízar-Vargas y Alfaro-Arce (2019) y Alpízar y Alfaro (2020), y evidencia un problema asociado a la formación inicial del personal docente de primaria.

Finalmente, el profesorado considera importante el acompañamiento por parte del MEP en los procesos de actualización, formación continua y otros apoyos curriculares.

Sobre este aspecto en particular, valoran que no es únicamente el MEP el que debe asumir los procesos de formación continua y que estos deben realizarse tanto en conocimientos matemáticos como en aspectos didácticos de la disciplina; así como en estrategias pedagógicas y evaluativas para la enseñanza de la matemática. Apuntan a que estas no sean teóricas, sino que implementen una metodología tipo taller donde la persona docente aprenda haciendo y pueda compartir con colegas.

Los programas de estudio de Matemática implicaron para todo el profesorado una serie de restos metodológicos, didácticos y evaluativos. Aunque se perciben esfuerzos por su implementación, según su fundamentación teórica, es claro que se siguen presentando dificultades.

Los hallazgos aquí presentados pretenden dar una guía para futuros procesos de investigación que permitan indagar, a nivel del aula, cuál es la realidad in situ sobre la implementación de los programas de estudio de matemática en la educación primaria. Por la situación del covid-19, la investigación realizada no pudo efectuar observaciones de aula, aunque estaban programadas.

La realidad de aula posterior al covid-19 podría mostrar otros resultados y evidenciar necesidades que serán importantes de analizar y atender. Es evidente que durante la pandemia las estrategias metodológicas y de evaluación sufrieron cambios con respecto a lo indagado en el estudio.

Lo que resulta evidente, a partir de los resultados obtenidos, es la necesidad de realizar procesos de formación continua que respondan a las necesidades del personal y que logren la mayor cobertura posible. Por distintas razones, no todo el profesorado de educación primaria posee las herramientas necesarias y suficientes para desempeñarse con éxito en la materia.

Esto, a la vez, es un llamado de atención a las instituciones formadoras de docentes de primaria, las cuales deben revisar sus planes de formación y actualizarlos con base en las políticas educativas actuales; señalamiento que coincide con lo indicado por Alpízar-Vargas y Alfaro-Arce (2019) y Alpízar y Alfaro (2020), como una forma de garantizar que la persona docente en formación adquiera las competencias básicas para desarrollar con éxito su labor educativa y las herramientas necesarias para continuar aprendiendo sobre la labor docente.

\section{Reconocimientos}

La información de este artículo se enmarca en el proyecto de investigación $L a$ enseñanza de las matemáticas en la educación primaria desde la perspectiva del docente: qué actitud posee y qué cree sobre la disciplina, cómo enseña los contenidos matemáticos y cómo se siente según su desempeño profesional, código SIA 0030-18, Universidad Nacional, Costa Rica.

\section{Conflicto de intereses}

Los autores declaran no tener algún conflicto de interés. 


\section{Declaración de la contribución de los autores}

Todos los autores afirmamos que se leyó y aprobó la versión final de este artículo. El porcentaje total de contribución para la conceptualización, preparación y corrección de este artículo fue el siguiente: R.G.A. 33,3\%, R.H.M. 33,3 \% y M.C.S. 33,3\%.

\section{Declaración de disponibilidad de los datos}

Los datos que respaldan los resultados de este estudio serán puestos a disposición por el autor correspondiente R.G.A, previa solicitud razonable.

\section{Referencias}

Alfaro, A. \& Alpízar, M. (2015). Docente de matemática: Perfil de salida de las universidades. Documento sin publicar del informe final del proyecto Hacia la utopía en la enseñanza de la Matemática en Educación Primaria, Escuela de Matemática, Universidad Nacional, Costa Rica.

Alfaro, A., Alpízar, M., Morales, Y., Ramírez, M. \& Salas, O. (2013). La formación inicial y continua de docentes de Matemáticas en Costa Rica. Cuadernos de Investigación y Formación en Educación Matemática, 8, 131-179. https://revistas.ucr.ac.cr/index.php/cifem/ article/view/12225

Alpízar, M., Barrantes, J., Bolaños, H., Céspedes, M., Delgado, E., Freer, D., Padilla, E. \& Víquez Ortiz, M. (2012). Aspectos relevantes sobre la formación docente en I y II ciclos en los temas probabilidad y estadística. $R e-$ vista Electrónica Educare, 16(2), 113-129. https://www.revistas.una.ac.cr/index.php/ EDUCARE/article/view/3934

Alpízar-Vargas, M. \& Alfaro-Arce, A. (2019). La formación universitaria de docentes de educación primaria: el caso de Matemáticas. Uniciencia, 33(2), 110-154. https://doi. org $/ 10.15359 /$ ru. $33-2.8$
Alpízar, M. \& Alfaro, A. (2020). Percepción de un grupo de docentes de educación primaria acerca de la preparación recibida durante su formación universitaria en cuanto al tema de las matemáticas. Actualidades Investigativas en Educación, 20(1), 1-31. https:// revistas.ucr.ac.cr/index.php/aie/article/ view/39978/40773

Alvarado, M. \& Vargas, M. (2020). Prácticas metodológicas y evaluativas empleadas por los docentes de primaria en un circuito de una Dirección Regional Educativa de Guanacaste, para la enseñanza de las Matemáticas y su relación con los lineamientos expuestos en los programas de estudios vigentes del Ministerio de Educación Pública de Costa Rica. [Tesis de Licenciatura]. Escuela de Matemática, Universidad Nacional, Costa Rica.

Castro, J. (2009). El maestro de educación primaria en la construcción de conceptos matemáticos. Memoria del X Congreso Nacional de Investigación Educativa, 1-12. http://www.comie. org.mx/congreso/memoriaelectronica/v10/ pdf/area_tematica_05/ponencias/0383-F.pdf

Chaves, E., Alfaro, A. \& Alpízar. M. (2015a). Percepción de los docentes de primaria, en ejercicio, acerca de las Matemáticas y su enseñanza en relación con los programas oficiales del MEP. Documento sin publicar del informe final del proyecto Hacia la utopía en la enseñanza de la Matemática en Educación Primaria, Escuela de Matemática, Universidad Nacional, Costa Rica.

Chaves, E., Alfaro, A. \& Alpízar. M. (2015b). Informe final del proyecto Hacia la utopía en la enseñanza de la Matemática en Educación Primaria. Escuela de Matemática, Universidad Nacional, Costa Rica.

Gaete, M. \& Jiménez, W. (2011). Carencias en la formación inicial y continua de los docentes y bajo rendimiento escolar en matemática en Costa Rica. Cuadernos de Investigación y Formación en Educación Matemática, 6(9), 93-117. http://revistas.ucr.ac.cr/index.php/ cifem/article/viewFile/6962/6648

Godino, J., Batanero, C. \& Font, V. (2003). Fundamentos de la enseñanza y el aprendizaje de las matemáticas para maestros. Departamento de Didáctica de la Matemática, Facultad de Ciencias de la Educación, Universidad de Granada. https://www.ugr.es/ jgodino/edumat-maestros/manual/1_Fundamentos.pdf 
Gonzaga, W. (2005). Las estrategias didácticas en la formación de docentes de educación primaria. Actualidades Investigativas en Educación, 5(1). https://www.redalyc.org/articulo. oa? id $=44750103$

Hernández, R., Fernández C. \& Baptista, P. (2014). Metodología de la Investigación (6ta ed). Mc-Graw Hill, México D.F.: México.

McMillan, J. \& Schumacher, S. (2005). Investigación educativa: una introducción conceptual. Madrid, España: Pearson Educación, S.A.

Ministerio de Educación Pública (2012). Programas de estudio de Matemáticas. San José, Costa Rica.

Otzen, T. \& Manterola, C. (2017). Técnicas de muestreo sobre una población a estudio. Int. J. Morphol., 35(1), 227-232. https://scielo.conicyt.cl/pdf/ijmorphol/v35n1/art37.pdf
Rodríguez, R. (2010). Habilidades docentes para la enseñanza de las matemáticas a nivel primaria bajo el enfoque por competencias. Boletín Electrónico de Investigación de la Asociación Oaxaqueña de Psicología A.C., 6(2), 337-345.

Ruiz, A. (2013). Reforma de la Educación Matemática en Costa Rica. Cuadernos de Investigación y Formación en Educación Matemática, 8. http://revistas.ucr.ac.cr/index.php/cifem/ issue/view/1186

Silva, M. (2008). La innovación en la enseñanza de las matemáticas en primaria: el modelo de matemáticas constructivas. Universidad Iberoamericana, México. https://docplayer. es/18949491-La-innovacion-en-la-ensenanza-de-las-matematicas-en-primaria-el-modelo-de-matematicas-constructivas.html

\section{(c) $\underset{\mathrm{BY}}{(1)}(\mathrm{NC})$}

La implementación de los programas de estudio de Matemática en primaria desde la visión de la persona docente (Ronny Gamboa-Araya $\bullet$ Randall Hidalgo-Mora $\bullet$ Mario Castillo-Sánchez) Uniciencia is protected by Attribution-NonCommercial-NoDerivs 3.0 Unported (CC BY-NC-ND 3.0) 